\title{
Crowdsourcing en bibliotecas
}

\author{
Leticia Paula Dobrecky \\ Centro de Documentación e Información Agropecuaria. Ministerio de Agroindustria, Argentina
}

\section{REVIEW}

\begin{abstract}
Resumen
En el presente trabajo se reflexiona sobre el crowdsourcing, término que acuñó Jeff Howe en el año 2006 y su relevancia para las bibliotecas. Si bien no existe consenso entre los autores para definir este término, se examinan los principales elementos que permiten describirlo y caracterizarlo junto con algunos ejemplos generales. Se analizan las razones para su aplicación en nuestro ámbito y algunas recomendaciones en base a experiencias de otras instituciones. Además, se describen algunos proyectos exitosos. Se discuten las oportunidades que presenta por el vínculo de pertenencia que se crea con el público, la posibilidad de cumplir con objetivos pendientes, el sentido de responsabilidad hacia las colecciones y un mayor acceso, así como los obstáculos centrados en la pérdida del control y de poder de los bibliotecarios frente a los prosumidores. Más allá de las amenazas que implica, es una herramienta con un gran potencial para que las bibliotecas tengan mayor visibilidad, se posicionen mejor en la sociedad y se asegure su perdurabilidad en el largo plazo.
\end{abstract}

Palabras clave

Crowdsourcing ; Bibliotecas ; Inteligencia colectiva ; Voluntarios

\section{Crowdsourcing in libraries}

\begin{abstract}
This article reflects on crowdsourcing, a term coined in 2006 by Jeff Howe, and its relevance to libraries. There is not a clear agreed definition. Thus, its main elements are examined to describe and characterize this phenomenon and a few examples are presented. The reasons to apply this tool in libraries, helpful tips and successful projects are analyzed. Engage users, add or enhance library collections, find solutions to problems, achieve libraries goals, create a sense of ownership are just some of the opportunities derived from this collaborative model. On the other hand, loss of power and control are threats that librarians must overcome. In conclusion, crowdsourcing represents an interesting challenge concept to increase the visibility of the library and assure longevity of the cultural heritage.
\end{abstract}

Crowdsourcing ; Libraries ; Wisdom of crowds ; Digital volunteers

\section{Crowdsourcing: origen y características}

El término "Crowdsourcing" se utiliza por primera vez en el año 2006 en un artículo publicado por Jeff Howe en la revista "Wired". La idea principal consiste en tomar una determinada tarea tradicionalmente desempeñada por un agente designado y externalizarla, realizando una convocatoria abierta a un amplio e indefinido grupo de personas. Desde otra perspectiva, implica rescatar los principios que funcionaron en los proyectos de software de código abierto y aplicarlos en todo el espectro del mundo de los negocios. Es un mecanismo para unir el talento y conocimiento que se encuentra disperso entre los seres humanos, con aquellos que lo necesiten.

Un antecedente al trabajo de Howe lo realizó James Surowiecki (2004), quien en su obra publicada, "The Wisdom of crowds", describe experimentos y eventos donde las actividades desempeñadas por el "público" sin ningún entrenamiento específico son realizadas tan bien o mejor que un único experto. Si bien, como explica Frederick Zarndt (2012), este fenómeno aparentemente no se plantea antes de dicho trabajo, el hecho en sí no es novedoso y se sustenta en la idea de "que dos cabezas piensan mejor que una". Si bien esa práctica de colaboración masiva, externalizada y de convocatoria abierta no es exclusiva de este siglo, lo que sí es 
novedoso, es el papel de las nuevas tecnologías digitales, Internet, las redes, dispositivos personales y la conectividad que permite el desarrollo, organización interna y distribución de tareas entre los miembros de las comunidades de interés que se generan (Lara, 2014).

No existe un acuerdo para definir al crowdsourcing sino una variedad de definiciones, que lo consideran desde distintos puntos de vista, que incluyen resolución de problemas o innovación aplicada a la mejora de los procesos de negocios. El análisis etimológico tampoco es de gran utilidad. Está conformado por dos términos: "crowd" (quienes participan en la iniciativa) y "sourcing" (prácticas de adquisición que apuntan a encontrar, evaluar e involucrar a proveedores de bienes y servicios). Por tales motivos, Enrique Estellés-Arolas y Fernando González-Ladrón-De-Guevara (2012a) centraron su análisis en la revisión de la literatura sobre el tema para identificar elementos comunes y así brindar una definición más acabada sobre esta idea. Para ello, se basaron en tres aspectos: el público, quién inicia el proyecto (crowdsourcer) y el proceso en sí mismo.

El público se perfila como un amplio grupo de individuos cuyas características en cuanto al conocimiento, número, heterogeneidad serán determinadas en función de los requerimientos de la iniciativa que se lleve a cabo. Tendrán que resolver un problema por medio de una tarea de variada complejidad lo que implicará la contribución voluntaria ya sea con su trabajo, dinero, conocimiento y/o experiencia. Quienes participan lo hacen motivados por una o más necesidades. Por dicha labor se puede recibir una compensación, no necesariamente económica, y siempre las define quien inicia el proyecto (crowdsourcer), que puede ser una compañía, institución, organización sin fines de lucro o un individuo. Por último, el crowdsourcing es un proceso participativo distribuido en línea.

Por tanto, se puede definir al crowdsourcing como un tipo de actividad participativa en línea, en la cual las instituciones o individuos proponen a un grupo de personas con conocimiento variado (amateurs y entusiastas) equipados con las herramientas correctas para la comunicación e intercambio de ideas, por medio de una convocatoria abierta y flexible, el desempeño de una tarea voluntaria. Esta interacción siempre implica un beneficio mutuo (Estellés-Arolas y González-Ladrón-De-Guevara, 2012a; Kowalska, 2012; Zarndt, 2012). Otra definición lo considera como un modelo de producción y resolución de problemas que utiliza la inteligencia colectiva de una comunidad en línea para servir a los objetivos específicos de la organización (Brabham, 2008).

El crowdsourcing se puede implementar como un potente instrumento para descubrir talentos, para impulsar proyectos o solucionar problemas que no se pueden abordar con los propios medios y recursos. Las motivaciones del público para participar en un proyecto de tal envergadura pueden ser diversas: aprender o profundizar en una temática, ganar experiencia, buscar reconocimiento, compartir conocimiento, identificarse con una idea o causa, desarrollar habilidades creativas, entretenimiento, altruismo, entre otras (Oomen y Aroyo, 2011; Ridge, 2014; Velasco, 2013; Zarndt, 2012).

Esta herramienta presenta diversas tipologías. En base a la naturaleza de las tareas a realizar por la multitud, Enrique Estellés-Arolas y Fernando González-Ladrón-De-Guevara (2012b), proponen una clasificación integradora: a) crowdcasting: quien inicia el proyecto plantea al público un problema o tarea, siendo recompensado quien lo resuelva antes o mejor; b) crowdcollaboration: considera a las iniciativas donde se produce una comunicación entre la multitud dejando relativamente al margen al "crowdsourcer". Normalmente no existe una recompensa económica; c) crowdcontent: el público aporta su mano de obra y su conocimiento para crear o encontrar contenido de diversa naturaleza. No implica una competencia. d) crowdfunding: un individuo o una organización buscan el financiamiento por parte de la multitud a cambio de una retribución; e) crowdopinion: se intenta conocer la opinión de los usuarios sobre un tema o producto. Dentro de algunas categorías se pueden localizar subtipos.

De todas las formas de crowdsourcing hay dos que están alcanzando un alto nivel de madurez: coworking o crowdworking (compartir lugares de trabajo en espacios colectivos) y el crowdfunding, donde el colaborador se convierte en socio, inversor o mecenas. Lo destacable y el motor de esta iniciativa es la aplicación de herramientas digitales para la gestión, viabilidad y transparencia del sistema de participación. (Lara, 2014).

Si bien el crowdsourcing surgió en el ámbito de los negocios, se puede implementar en diversas esferas dada su flexibilidad, como ser las actividades sociales, ciencia, en la industria, incluyendo arquitectura y planeamiento urbano, avisos sobre tecnología, diseño gráfico, entre otros. También hay que considerar a las organizaciones sin fines de lucro.

Desde el ámbito empresarial, existen varios casos de crowdsourcing. La compañía Lego (https://ideas.lego.com/) lanzó una campaña donde el público participa presentando su propio diseño. El que 
obtiene más votos (debe llegar a 10.000) pasa a la etapa de revisión. Se elige al ganador y se fabrica el producto para la venta. Otro caso "Amanzon's Mechanical Turk" (http://www.mturk.com), propone desarrollar microtareas (alta modularidad) que implican poco tiempo y se ofrece a cambio una retribución económica que en general es mínima. Se presenta como un mercado de trabajo donde se requiere de la inteligencia humana para la identificación de objetos en una foto o video, traducción de pasajes de textos en otros idiomas, transcripción de registros de audio, categorización de sitios web, etc. Justamente, este proyecto se basa en la idea de que existen tareas que los seres humanos desarrollan más efectivamente que las computadoras. Probablemente es un claro ejemplo de una plataforma construida alrededor de interacciones que se basan en motivaciones extrínsecas (Oomen y Aroyo, 2011)

"Old Weather" (http://www.oldweather.org/) es un proyecto que se enmarca dentro de Zooniverse, una red de científicos-ciudadanos, que tiene como objetivo producir investigación que sólo se puede realizar gracias a la contribuciones del público. La idea consiste en transcribir las bitácoras de las embarcaciones de Estados Unidos desde mediados del siglo XIX. Con esta información se contribuirá con los modelos de proyección del tiempo y mejorará el conocimiento sobre las condiciones históricas del medio ambiente. Los historiadores podrán descubrir los movimientos de los barcos y las historias del personal a bordo.

Las instituciones que resguardan el patrimonio cultural poseen una larga tradición de colaboración con el público. También han visto en estas dinámicas un medio para movilizar a sus públicos de interés y generar mayor participación en sus causas (Lara, 2014). En este contexto, es importante pensar en el crowdsourcing como una oportunidad para proveer a los individuos caminos significativos para comprometerse y contribuir a la memoria pública (Owens, 2013). Siguiendo esta línea de trabajo, surge el Crowdconsortium (http://www.crowdconsortium.org/), una organización dedicada a apoyar la investigación, impulso y desarrollo de buenas prácticas en el ámbito de los archivos, museos y bibliotecas.

El Instituto Smithsonian (https://transcription.si.edu/) impulsa un programa de voluntarios denominado "Digital Volunteers: transcription Center", para contribuir con la transcripción de documentos históricos y datos de biodiversidad. El objetivo es hacer más accesible y útil la colección sobre arte, ciencia, historia, para los curadores, investigadores y el público en general. Las computadoras tienen dificultad para interpretar el material manuscrito. Por ello, muchos conocimientos y secretos contenidos en sus páginas pueden salir a la luz con la contribución de los participantes en diversos proyectos. Se trata de lograr un balance entre la calidad y la velocidad en el proceso.

"Describe me" (http://describeme.museumvictoria.com.au/) es un proyecto impulsado por el Museo Victoria de Australia para hacer de la colección en línea un recurso más útil y accesible para las personas no videntes o con capacidad visual disminuida. El público participa elaborando una descripción breve de lo que se ve en las imágenes.

Con el auge de los medios sociales, los usuarios tienen una doble condición: consumen y crean contenidos, convirtiéndose en prosumidores. Las bibliotecas no son ajenas a este escenario dinámico donde la participación y el trabajo en colaboración son dos ejes fundamentales. En este marco, el crowdsourcing se posiciona como una herramienta para brindar nuevos caminos que permiten establecer un vínculo e intercambio más enriquecedor con el público. Además favorece la consecución de proyectos cuando se cuenta con un presupuesto reducido. Sin embargo, implica implementar cambios drásticos en los flujos de tareas y especialmente en la mentalidad de los bibliotecarios.

El propósito del presente trabajo es examinar las oportunidades y amenazas que presenta el crowdsourcing en las bibliotecas. Para ello, se parte de una revisión de la bibliografía sobre el tema donde se identifican las distintas posturas argumentales de los expertos en la materia así como también se estudian algunos casos en los cuales su aplicación fue exitosa. El mayor caudal de información se localiza en artículos de revistas y en presentaciones a congresos, abarcando desde el año 2008 aproximadamente hasta el 2015.

\section{El crowdsourcing en las bibliotecas}

Existen varios ejemplos de aplicación de crowdsourcing en bibliotecas y en otras instituciones vinculadas con el patrimonio cultural como archivos y museos. Algunas de las principales iniciativas están relacionadas con la transcripción de textos, identificación de imágenes y otros contenidos, etiquetado de elementos en los documentos digitalizados a través de plataformas específicas, detección de errores en los catálogos, agregado de información a los registros, creación de e-books, diseño de un nuevo servicio de referencia, descripción de 
material que se encuentra pendiente de ser ingresado por falta de tiempo, verificación de nombres en el catálogo de autoridad (Barttlet, 2014; Holley, 2009; Oomen y Aroyo, 2011).

En el ámbito de las bibliotecas universitarias existen plataformas que permiten a los investigadores gestionar, compartir y comentar resultados de sus trabajos como publicaciones, bibliografía de respaldo, consultas, flujos de tareas experimentales. Desde el punto de vista del crowdsourcing, el elemento clave en estas iniciativas es la fuerte comunidad en línea. El principal incentivo para registrarse y utilizar dichos espacios es el servicio que proveen. Otra aplicación está orientada a crear grupos de interés sobre temas científicos particulares. Aquí también se incluyen los proyectos centrados en investigación y desarrollo (innovación abierta). Por último, la tercera área es muy amplia y abarca diferentes tareas donde son necesarias las habilidades técnicas de las personas ya que algunas funciones no son desempeñadas eficientemente por las computadoras (Simperl, 2015).

Entre las principales razones para aplicar el crowdsourdcing, Kathryn Eccles (2015) enumera las siguientes: agrega contenido y valor, analiza grandes conjuntos de datos, fomenta la participación, transforma el acceso a los recursos, crea o amplía las redes, educa, permite nuevas preguntas de investigación.

Una de las instituciones pioneras en la aplicación de crowdsourcing para la corrección de textos es la Biblioteca Nacional de Australia (http://www.nla.gov.au/). En el año 2007, comenzó un programa de digitalización de periódicos que datan desde 1803 para el acceso y la preservación. El problema radica en que la tecnología OCR brinda resultados pobres e imprecisos en el caso de los materiales antiguos. En el 2008 se lanzó al público una versión beta del servicio y en el año 2010 se integró a Trove, un sistema que permite la búsqueda a través de esos textos traducidos electrónicamente. Por tal motivo, es fundamental la participación del público en las correcciones para mejorar la búsqueda y recuperación.

Otros casos exitosos de implementación están dados en Estados Unidos. La New York Public Library lanza en el 2011 el proyecto denominado "What's on the menu?" (http://menus.nypl.org/). La actividad se centra en la transcripción de los menús de restaurantes que datan desde 1840 hasta la fecha. Esta enorme colección reúne datos muy valiosos (platos, precios, organización, historia y cultura de las comidas) y es consultada por historiadores, chefs, novelistas y toda persona entusiasta de esta temática. Si bien la digitalización junto al OCR es beneficiosa para la búsqueda, presenta algunos inconvenientes para los materiales manuscritos y aquellos con una tipografía extravagante. Para lograr su objetivo, cuentan con la ayuda de voluntarios que colaboran con la construcción de una verdadera base de datos de comidas.

La biblioteca de la Universidad McMaster (https://postcards.mcmaster.ca/) ubicada en Canadá, presenta un proyecto de crowdsourcing para que el público colabore en la descripción de postales históricas que fueron adquiridas a un coleccionista privado en el 2008. Una vez concluida la identificación de cada una de ellas, se incorporan al Archivo Digital. De este modo los usuarios pueden acceder y conocer cómo lucían las ciudades canadienses y de otras partes del mundo hace ochenta o cien años atrás. Además, es de gran utilidad para que los investigadores localicen material valioso.

La British Library junto con otras instituciones (National Trust, National Trust for Scotland y AudioBoom) desarrolló "Sounds of our shores", un mapa interactivo que reúne tantos sonidos como sean posibles de todo el paisaje costero del Reino Unido durante el verano del 2015. Se invitó al público a subir su registro marítimo favorito (aves, mar, estuarios, playas, puerto, etc.) a través de una aplicación o del navegador. De este modo, se crea un recurso digital permanente que intenta reflejar la diversidad de la zona y el importante rol que juega en la vida cotidiana.

En España, la Biblioteca de Catalunya lanza el proyecto "Transcriu-me!! (http://transcriu.bnc.cat/) para mejorar el acceso a los contenidos digitales. En el 2014 y a través del crowdsourcing (o participación en línea ciudadana), la Filmoteca propone transcribir un millar de carteles con información muy valiosa sobre la programación cinematográfica de comienzos del siglo XX. Esta iniciativa es fruto de la colaboración de la Institución y el CSUC (Consorci de Serveis Universitaris de Catalunya). Existen otros proyectos en curso como la obra literaria manuscrita del autor Àngel Guimerà y "Testimonis bibliotecaris" (colección de diarios en los cuales los bibliotecarios registraban la labor cotidiana, desde la creación de la Red de Bibliotecas Populares de la Mancomunitat de Catalunya en los albores del siglo XX).

El crowdsourcing alienta a los usuarios a participar en la generación de conocimiento con otros miembros de la comunidad académica. Se presenta como un modelo efectivo para incorporar nuevas ideas y perspectivas. Basado en esta práctica se desarrolló un sistema de referencia denominado "CrowdAsk" de la Purdue University 
Libraries. La idea es proveer información de mayor utilidad a los estudiantes (que muchas veces buscan ayuda entre sus pares o en los consejeros académicos) y cumplir con el rol de formación. El sistema permite ingresar preguntas y respuestas relacionadas con la biblioteca, sus servicios, asesoramiento. La ayuda la reciben no sólo por parte de los bibliotecarios sino de investigadores con experiencia e intereses compartidos. De este modo, se convierte en una comunidad en línea y una fuente de consulta permanente (Stonebraker y Zhang, 2015).

Para implementar exitosamente proyectos de crowdsourcing es recomendable analizar algunas cuestiones claves. En principio, el objetivo de la iniciativa debe estar claramente definido y destacado en el sitio web y debe representar un desafío. Asimismo, los avances de las tareas se deben presentar en una tabla para que los participantes conozcan el progreso alcanzado (la comunidad siempre debe conocer el punto alcanzado). Otras variables a considerar son la usabilidad, la velocidad del sistema, que sea intuitivo y sencillo.

Con relación a las actividades propuestas tienen que ser entretenidas, diversas y sencillas para que cada persona pueda encontrar aquella que le atraiga y que conecte con sus habilidades e intereses, de modo que pueda aportar lo mejor de sí. Los resultados deben ser transparentes y visibles. Además, se debe permitir que los voluntarios se identifiquen y puedan ocupar un lugar destacado. Es fundamental implementar vías de interacción dinámica, saber escuchar y adoptar una actitud positiva en cuanto a la labor realizada. Otro aspecto que se debe planificar es el denominado sistema de retribuciones que pueden ser materiales con retorno económico o intangibles teniendo en cuenta las motivaciones que pueden movilizar a los participantes (Holley, 2009; Lara, 2014).

\section{Oportunidades vs amenazas}

La mayoría de las instituciones no cuentan con los recursos financieros, humanos, o con el tiempo suficiente para cumplir con los objetivos propuestos. El crowdsourcing se posiciona como una práctica para que el público participe y se involucre. Les brinda una vía para hacer algo más que sólo consumir información. Esto genera un vínculo de pertenencia, idea de "propiedad" y sentido de responsabilidad hacia el patrimonio. Además, se crean nuevas comunidades virtuales, se agrega valor a los datos, se construye y profundiza la confianza de los usuarios hacia la misma. Justamente, las fortalezas están dadas en que son un terreno fértil para la puesta en marcha de estos proyectos ya que tienen experiencia en captar voluntarios y son confiables (Bartlet, 2014; Ellis, 2014, Holley, 2010; Kowalska, 2012; Owens, 2013; Zastrow, 2014).

En la mayoría de los casos, el público que colabora en proyectos de crowdsourcing lo hace voluntariamente sin recibir una retribución económica. Esto representa una gran ventaja para muchas bibliotecas que cuentan con un reducido presupuesto. Sin embargo, esto no descarta el diseño de un sistema de recompensas y premios que pueden estar dados por un reconocimiento social y hasta material (Holley, 2009; Lara, 2014). En el sector privado, especialmente, se plantea un dilema ético ya que las empresas ofrecen micropagos por las actividades desempeñadas, pero su ganancia es mucho mayor que su inversión. De todos modos, este aspecto no impacta tanto en las instituciones culturales porque están posicionadas como un bien público. Por ello, los voluntarios pueden estar seguros que su contribución no será explotada con fines comerciales (Ellis, 2014). Es un camino para que participen los usuarios y los ciudadanos amateurs (en el sentido positivo del término) en la creación y desarrollo de contenidos (Owens, 2013).

Otro aspecto a considerar son las implicaciones que adquieren los derechos de autor en las contribuciones de los usuarios (si son originales o modifican obras previas) y las responsabilidades de las partes involucradas. Por ello, es fundamental diseñar un sistema de garantías que equilibre los derechos en un contexto de cultura digital (Lara, 2014).

Las bibliotecas ganaron su reputación preservando la calidad y veracidad de la información que ofrecen teniendo control sobre todo el proceso. En los proyectos de crowdsourcing se debe considerar el error humano y el tiempo que se consume para las correcciones. Es fundamental establecer sistemas de control de calidad. Otra problemática se genera por la pérdida de poder que sienten los bibliotecarios en este contexto. En este sentido, Rose Holley (2010), explica que al permitir que el público (con o sin conocimiento especializado) participe en la creación de metadatos, contenido u otra tarea, se puede percibir como una forma de sustitución de la pericia de los profesionales y del paradigma centrado en expertos.

Los proyectos de crowdsourcing necesitan de recursos materiales y humanos lo que trae aparejado costos de organización y coordinación. Primeramente, hay que considerar la infraestructura tecnológica (software y el hardware), el soporte, Internet y las comunicaciones. Además, hay que tener en cuenta el diseño de una mesa de ayuda para los voluntarios (Help Desk), elaboración de tutoriales y redacción de documentación relacionada 
con buenas prácticas. Por otro lado, la/s persona/s responsables o gestores deben reunir condiciones para un liderazgo firme, visión, habilidades de negociación y perseverancia. Los voluntarios deben sentirse valorados y saber que su contribución marca una diferencia y que el tiempo dedicado está bien aprovechado, (Ellis, 2014; Lara, 2014; Zarndt, 2012).

\section{Un nuevo desafío}

El crowdsourcing se presenta como un fenómeno con gran potencial para que las bibliotecas se posicionen mejor en la sociedad. Queda establecido que la mayoría de las instituciones poseen problemas presupuestarios y escaso personal. Esta práctica flexible permite implementar proyectos ambiciosos con un impacto positivo en la comunidad al generarse nuevos vínculos y mayor acceso a las colecciones.

El mayor desafío que se plantea es para los profesionales ya que implica un cambio de mentalidad, superando el temor a la pérdida de control y poder. Este nuevo escenario conlleva modificar el flujo de tareas para incorporar al nuevo recurso humano, ansioso por intervenir, pero que es necesario guiar, capacitar, gestionar y escuchar. Es fundamental, enfatizar la comunicación entre todos los actores del proceso. Además, es necesario establecer mecanismos de control de calidad que no vayan en desmedro de los vínculos que se establezcan con la comunidad de usuarios.

Por último, más allá de los obstáculos que presenta el crowdsourcing, posee grandes posibilidades para que las bibliotecas tengan una mayor visibilidad, se generen nuevos productos y servicios gracias al trabajo colaborativo, se fomente la cooperación y se asegure su perdurabilidad en el largo plazo.

\section{Referências}

Bartlett, J. (2014). Crowdsourcing in libraries and archives. Kentucky Libraries, 78(2), pp.6-8.

Brabham, D. (2008). Crowdsourcing as a model for problem solving: an introduction and cases. Convergence, 14(1). Recuperado de http://www.crowdsourcingverband.de/verband/download/

Eccles, K. (2015, 12 Agosto). What is crowdsourcing? En Crowdsourcing the Library and Archive. ALLISS Conference. Recuperado de http://www.slideshare.net/alissinfo/what-is-crowdsourcing-51635905

Ellis, S. (2014). A history of collaboration, a future in crowdsourcing: positive impacts of cooperation on British Librarianship. Libri. 64(1), pp.1-10.

Estellés-Arolas, E. y González-Ladrón-De-Guevara, F. (2012a). Towards an integrated crowdsourcing definition. Journal of Information Science, 38(2), pp.189-200.

Estellés-Arolas, E. y González-Ladrón-De-Guevara, F. (2012b). Clasificación de iniciativas de crowdsourcing basadas en tareas. El Profesional de la Información, 21(3). Recuperado de http://dx.doi.org/10.3145/epi.2012.may.09

Holley, R. (2009, 18 noviembre). Crowdsourcing and social engagement: potential, power and freedom for librarian and users. Trabajo presentado en Annual meeting - Pacific Rim Digital Library Alliance, Auckland. Recuperado de http://pr-rla.org/2009/10/crowdsourcing-and-social-engagement/

Holley, R. (2010). Crowdsourcing: How and Why Should Libraries Do it? D-Lib Magazine, 14(3/4). Recuperado de http://www.dlib.org/dlib/march10/holley/03holley.html

Howe, J. (2006). "The rise of Crowdsourcing". Wired, 14(66).

Kowalska, M. (2012). Crowdsourcing in libraries. Recuperado de http://eprints.rclis.org/18807/

Lara, T. (2014). Crowdsourcing: cultura compartida. En Acción Cultural Española. Anuario AC/E de Cultura Digital. Recuperado de http://www.accioncultural.es/es/publicacion digital anuario ac e cultura digital focus 2014

Owens, T. (2013). Digital Cultural Heritage and the Crowd. Curator: The Museum Journal, 56(1), pp.121-130. 
Oomen, J. y Aroyo, L. (2011). Crowdsourcing in the Cultural Heritage Domain: opportunities and challenges. En Proceedings of the 5th International Conference on Communities and Technologies (C\&T '11). ACM, New York, USA.

Ridge, M. (2014). Crowdsourcing 101: fundamentals and case studies. Recuperado de https://www.webjunction.org/events/webjunction/crowdsourcing-101.html

Stonebraker, I. y Zhang, T. (2015). Crowdsourcing Reference Help: using technology to help users help each other. En: ACRL Proceedings. Recuperado de http://www.ala.org/acrl/acrl/conferences/acrl2015/papers

Simperl, E., (2015). How to Use Crowdsourcing Effectively: Guidelines and Examples. Liber Quarterly. 25(1), pp.18-39.

Surowiecki, J. (2004). "The Wisdom of Crowds”. New York: Anchor Books.

Velasco, J. (2013, febrero 7). Crowdsourcing: la colaboración como motor de las grandes ideas. [Mensaje en Blog]. Recuperado de http://blogthinkbig.com/crowdsourcing-colaboracion-motor-ideas/

Zarndt, F. (2012). Putting the world's cultural heritage online with crowd-sourcing. En IFLA Pre-Conference, Finlandia. Recuperado de http://www.ifla.org/node/8154

Zastrow, J. (2014). The Digital Archivist: Crowdsourcing cultural heritage. Computers in Libraries, 34(8). Recuperado de http://www.infotoday.com/cilmag/oct14/index.shtml

\section{Datos del autor}

\section{Leticia Paula Dobrecky}

Licenciada en Bibliotecología y Ciencia de la Información egresada de la Facultad de Filosofía y Letras de la Universidad de Buenos Aires (UBA). Adscripta a la Cátedra de Fuentes de Información en Humanidades y Ciencias Sociales de la UBA (2002-2003). Area de Procesos Técnicos del Centro de Documentación e Información del Ministerio de Economía y Producción (2003). Desde esa fecha hasta la actualidad, área de Referencia del Centro de Documentación e Información Agropecuaria del Ministerio de Agroindustria. Idobre@magyp.gob.ar

Recibido - Received: 2016-03-04

Aceptado - Accepted: 2016-04-12

\section{(cc) $\mathrm{EY}$}

This work is licensed under a Creative Commons Attribution 4.0

United States License.

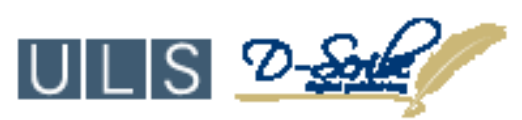

This journal is published by the University Library System of the University of Pittsburgh as part of its D-Scribe Digital Publishing Program and is cosponsored by the University of Pittsburgh Press. 